\title{
Trehalose drastically extends the in vitro vegetative culture period and facilitates maintenance of Torenia fournieri plants
}

\author{
Hiroyasu Yamaguchi ${ }^{\dagger}$, Katsutomo Sasaki ${ }^{\dagger}$, Masahito Shikata, Ryutaro Aida, \\ Norihiro Ohtsubo* \\ National Institute of Floricultural Science, National Agriculture and Food Research Organization, Tsukuba, Ibaraki \\ 305-8519, Japan \\ *E-mail: nohtsubo@affrc.go.jp Tel \& Fax: +81-29-838-6822
}

Received December 26, 2010; accepted January 24, 2011(Edited by T. Terakawa)

\begin{abstract}
While torenia (Torenia fournieri Lind.) is a useful model flower for molecular biological studies of floral architecture, the maintenance of plant materials and resulting transgenic plants requires vegetative propagation due to its heterozygous nature. Reduction of labor and costs for maintaining thousands of in vitro torenia cultures is therefore a critical issue. We found that substituting trehalose for sucrose drastically extended the culture period to 70 days, which is more than twice as long as for the common, sucrose-based medium, without reduction in plant viability. Comparative measurement of the plant mass indicated that the increased survival benefit of the trehalose-based medium might be on account of improvement in the rhizosphere environment through reduction of root density in the culture, rather than by reduced plant growth. No harmful effects arising from the trehalose-based medium were observed in 1,800 laboratory lines during the bimonthly subculture for over 12 months, except for a wilting on the first transfer to the trehalose-based medium. In conjunction with the use of the commercial food additive, Okome-ni-TREHA ${ }^{\circledR}$ rather than reagent-grade trehalose, we have succeeded in reducing the costs and labor associated with the culture medium to less than one third of those for the sucrose-based system.
\end{abstract}

Key words: In vitro culture, rhizosphere environment, Torenia fournieri, trehalose.

Commercially important flowers such as chrysanthemums, carnations, and roses are heterozygotes; hence maintenance of stable varieties by seed propagation is impossible. Furthermore, inbreeding depression as a result of repeated selfing in some species causes difficulty in breeding pure lines. Therefore, maintenance of these flowers requires vegetative propagation.

Although torenia is a heterozygous plant, it is useful for genetic engineering because of its suitability for tissue culture, simplicity of transformation, and short generation time (Aida 2008). We have produced vast numbers of transgenic torenia (Narumi et al. 2008, 2011; Sasaki et al. 2010, 2011; Shikata et al. 2011) and ion beam-irradiated torenia mutants (Sasaki et al. 2008) in order to generate novel floral traits and thus study phenotypic behavior, while routinely maintaining 1,800 important lines by in vitro culture. Because the monthly subculture of these plants is costly and laborious, technical developments to extend the culture period have been awaited. The standard culture period for general, sucrose-based medium has been empirically determined to be between 25 and 30 days to keep all plants in a healthy condition. Torenia plants at the end of the culture period usually reach to the top of the vessels, and continuous culture significantly decreases plant viability through rapid browning and wilting.

Sucrose is the most frequently used carbon source for in vitro-cultured plants, including torenia (Aida and Shibata 2001), but the effects of trehalose on in vitro culture have now been investigated in several plants. Liu et al. (2006) reported that trehalose was effective for propagation of Phalaenopsis and Doritaenopsos protocorm-like bodies. Furthermore, jojoba (Simmondsia chinensis) treated with trehalose exhibited greater adaptability to environmental conditions during acclimatization (Llorente et al. 2007). However, reports on the utilization of trehalose for extension of the culture period are absent. In this study, we report that the addition of trehalose to the culture medium rather than sucrose extends the culture period of in vitro torenia vegetative culture by more than twofold.

In this study, Torenia fournieri Lind. 'Crown Violet' was used as the plant material. The stem pieces $2-3 \mathrm{~cm}$ in height with some leaves and axillary buds were excised from torenia plants cultured for 25 days on $1 / 2$

Abbreviations: MS; Murashige and Skoog.

${ }^{\dagger}$ The authors contributed equally to this work.

This article can be found at http://www.jspcmb.jp/ 
strength Murashige and Skoog medium (1/2 MS, pH 5.7) (Murashige and Skoog 1962) containing 3.0\% (w/v) sucrose (Wako, Osaka, Japan) and $0.2 \%(\mathrm{w} / \mathrm{v})$ gellan gum (Wako). Four pieces of each excised plant were then transferred to the culture vessels (CP-M-1, Iwaki, Tokyo, Japan), each containing $50 \mathrm{ml}$ of $1 / 2 \mathrm{MS}$ medium $(\mathrm{pH}$ 5.7) with $0.2 \%(\mathrm{w} / \mathrm{v})$ gellan gum and either $3.0 \%(\mathrm{w} / \mathrm{v})$ trehalose dihydrate (Wako) or $3.0 \%(\mathrm{w} / \mathrm{v})$ sucrose. The plants were cultured in a growth chamber maintained at $25^{\circ} \mathrm{C}$ under fluorescent light $(16 \mathrm{~h}$ light $/ 8 \mathrm{~h}$ dark, $\left.85 \mu \mathrm{mol} \mathrm{m}^{-2} \mathrm{~s}^{-1}\right)$. Seventy days after subculture, torenia plants grown on the sucrose-based medium turned brownish, in particular around the basal parts of the stems and roots (Figure 1A, left), and the culture medium exhibited browning, probably because of accumulation of waste products such as phenolic compounds (Figure 1B, left). In contrast, torenia plants subcultured on the trehalose-based medium remained healthy (Figure 1A, right), and no browning of the culture medium was observed (Figure 1B, right). Therefore, application of trehalose to the culture medium rather than sucrose extended the culture period more than twofold, thereby reducing the labor input required for subculturing.

After 30 days of culture, the root density in trehalosecultured plants was lower than that in sucrose-cultured
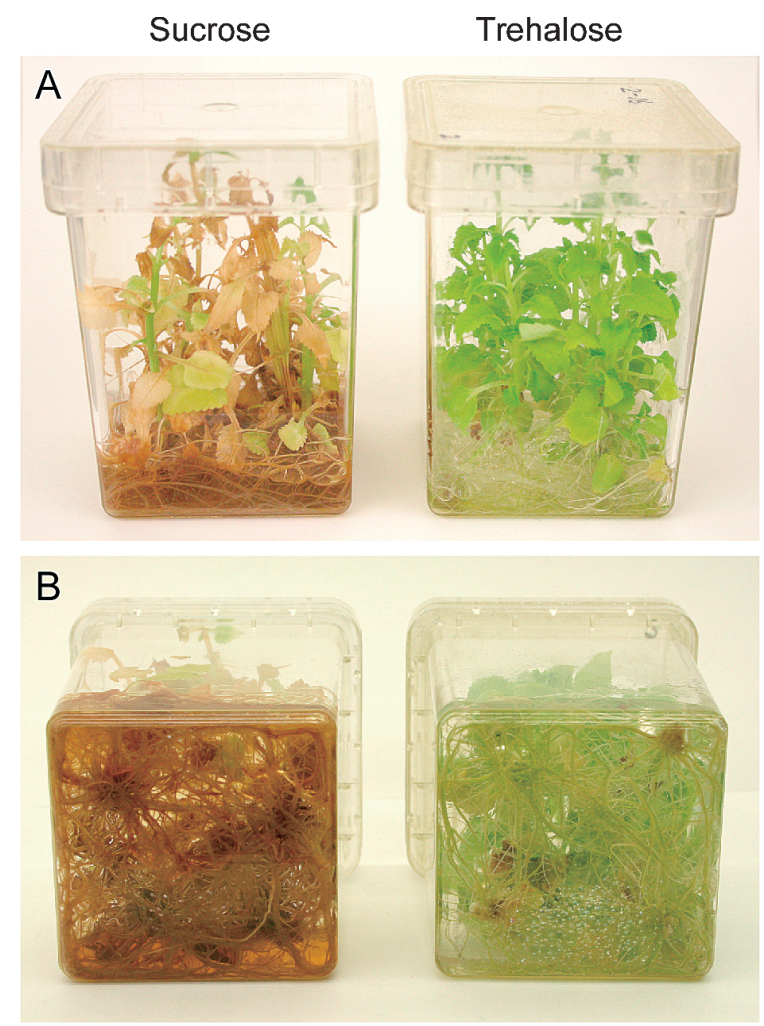

Figure 1. Effect of trehalose application on in vitro-cultured torenia. Excised stem pieces were cultured in either a sucrose-based medium (left) or a trehalose-based medium (right) for 70 days; (A) side view, (B) bottom view.

plants (Figure 2). To understand the physiological role of trehalose in extending the culture period, we performed a comparative experiment on plant weight by focusing on the difference in root growth. The fresh weight of the aerial parts (leaves and stems) and roots at 30 days after subculture were measured for 15 torenia plants (Table 1). Different increments of plant growth were observed between sucrose- and trehalose-cultured plants, although this difference did not show any statistical significance. As observed in Table 1, the total average weight of trehalose-cultured plants $(1146.5 \mathrm{mg})$ was $24 \%$ lower than that of sucrose-cultured plants $(1503.4 \mathrm{mg})$. In addition, the roots:aerial parts ratio in the trehalosegrown plants $(0.297)$ was $17 \%$ lower than that in the sucrose-grown plants $(0.356)$. These differences reflected our observations on the 12-month maintenance of in vitro-cultured torenia, and this result indicates that reduction in total plant mass and relative root mass may synergistically extend the culture period of trehalose-cultured plants, probably by reducing root density and improving the rhizosphere environment. Similar inhibition of root elongation was observed in Arabidopsis when sucrose-grown plants were transferred to a trehalose-based medium (Bae et al. 2005; Wingler et al. 2000). Furthermore, Llorente et al. (2007) reported that addition of trehalose to a sucrose-based medium decreased root development in jojoba. Trehalose also reduced root mass in torenia in our study, and may have influenced growth inhibition in the aerial parts. In our

Sucrose

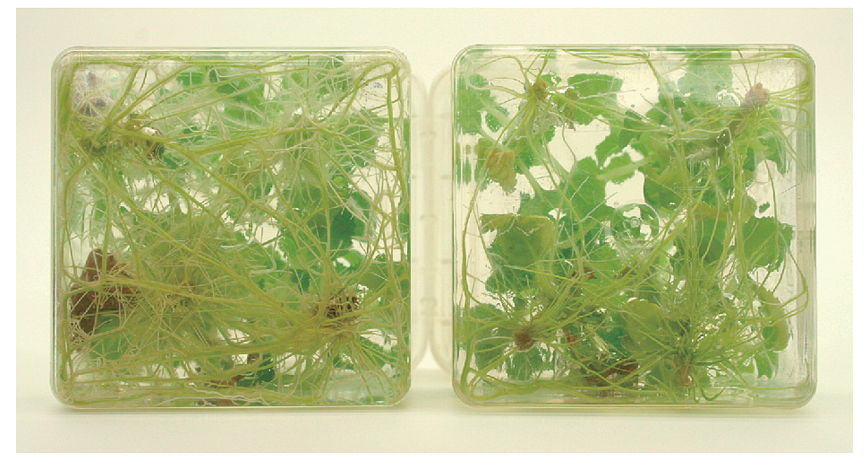

Figure 2. Effect of trehalose application on root density of cultured torenia. Roots after 30 days' subculture in media containing either sucrose (left) or trehalose (right).

Table 1. Differences in growth in T. fournieri on trehalose- and sucrose-based media

\begin{tabular}{lcc}
\hline Carbon source & $\begin{array}{c}\text { Fresh weight of leaf } \\
\text { and } \text { stem }^{\mathrm{a}}(\mathrm{mg})\end{array}$ & $\begin{array}{c}\text { Fresh weight of } \text { root }^{\mathrm{a}} \\
(\mathrm{mg})\end{array}$ \\
\hline Trehalose 3\% & $884.3 \pm 114.4$ & $262.2 \pm 70.8$ \\
Sucrose 3\% & $1108.8 \pm 103.6$ & $394.6 \pm 56.9$ \\
\hline
\end{tabular}

${ }^{\text {a }}$ Data are mean \pm S.E. $N=15$

Fresh weights were investigated 30 days after planting on each medium. 


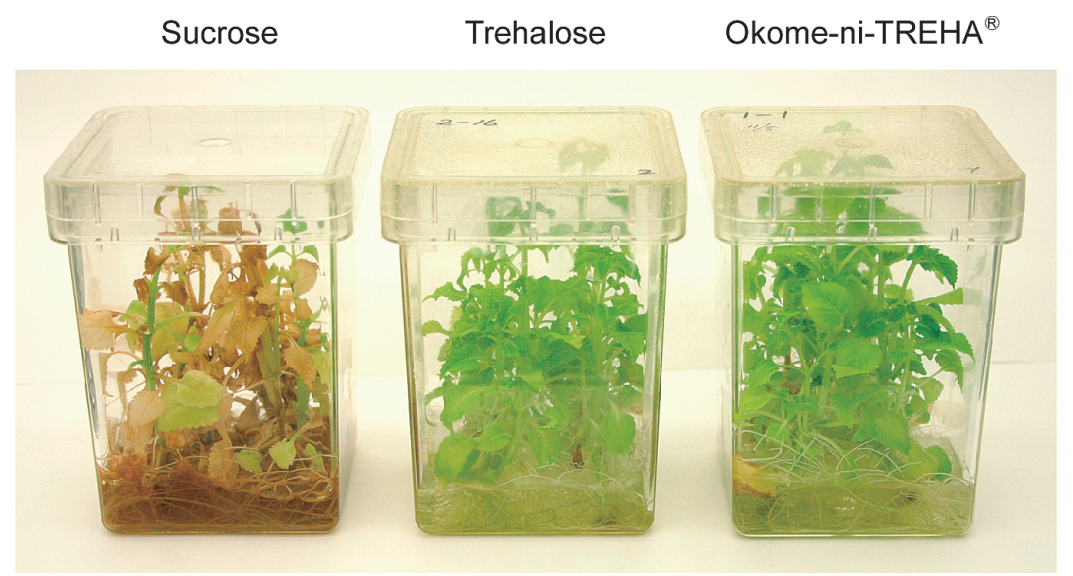

Figure 3. Comparison between the effects of trehalose reagent and Okome-ni-TREHA ${ }^{\circledR}$ on in vitro-cultured torenia. Excised stem pieces were cultured in a sucrose-based medium (left), a trehalose reagent-based medium (center), and a Okome-ni-TREHA ${ }^{\circledR}$-based medium (right) for 70 days.

study, however, the reduction in total mass may not be a major factor for extension of the culture period because the trehalose-cultured plants maintained the same condition even after the total mass had been increased to the same level as the upper limit of sucrose-cultured plants, and they survived for over 70 days (data not shown). Trehalose not only inhibited root growth in cultured torenia, but it is also possible that it increased abiotic stress tolerance (Iordachescu and Imai 2008) and contributed to enhanced survival. In the future, we would undertake further study on the mechanisms of extension of the culture period and growth reduction in trehalosebased media.

As briefly mentioned above, wilting was observed in up to $25 \%$ of plants just after the first transfer to the trehalose-based medium (data not shown). At this stage, most of these plants do not grow well and wilt within 20 days after transfer. Because this is observed only after replacement of the carbon source from sucrose to trehalose and is never observed after the second transfer to the same trehalose medium, it is assumed that acclimatization to trehalose must have stressed the plants to some extent. This wilting phenomenon is seen to a greater extent in chrysanthemum (Chrysanthemum morifolium Ramat. 'Sei-marine', data not shown), but hardly observed in Japanese gentian (Gentiana triflora $\times$ G. scabra 'Albireo' and 'Polano White'; M. Nishihara, personal communication). Estimation of adaptability and improvement through reduction of this transient stress will be a key factor in application of trehalose to other plant species. We are now investigating the cause of this phenomenon.

In terms of cost-effectiveness, reagent grade trehalose is too expensive and is unsuitable for routine culture; therefore, we examined the alternative product Okomeni-TREHA ${ }^{\circledR}(\mathrm{H}+\mathrm{B}$ Life Science, Okayama, Japan), which consists of over $98 \%$ (dry matter) enzymatically synthesized trehalose and a small amount of unreacted glucose. Okome-ni-TREHA ${ }^{\circledR}$ is available at less than half the cost of standard sucrose used in routine culture. After 2 years of verification experiments with bimonthly subculture, we confirmed that Okome-ni-TREHA ${ }^{\circledR}$ is equally effective in extension of the culture period as reagent grade trehalose (Figure 3), and we now apply Okome-ni-TREHA $^{\circledR}$ to all our laboratory lines as a substitute for trehalose. Okome-ni-TREHA ${ }^{\circledR}$ contains a small amount of unreacted glucose; however, this foreign substance did not affect the maintenance of in vitrocultured torenia. In fact, no harmful effects have been observed over the past 12 months, apart from wilting on the first transfer from sucrose- to trehalose-based medium, as described above. Furthermore, trehalose culture has no discernible impact on any of the events involved in Agrobacterium-mediated transformation, at least in torenia (Supplementary Table S1). Together with the extended culture period, cost of this culture medium is around $25 \%$ of that of the previous sucrose-based system.

Trehalose-based culture has an additional advantage in the facilitation of transgenic plant production over conventional, Agrobacterium-mediated method in torenia. In the previous sucrose-based system (Aida and Shibata 1995), monthly subculture of weekly isolated transgenic adventitious shoots was required for approximately 2 months to ensure that sufficient numbers of transgenic torenia grew enough size for naturalization. In addition, these shoots could not be naturalized simultaneously. In contrast, shoots in the trehalose-based medium can be continuously cultured until naturalization without the need for subculture, allowing naturalization en masse (Supplementary Figure S1). Thus, utilization of trehalose contributes not just to a reduction in labor and costs but also to the acceleration of research in torenia by simplifying transformation. 


\section{Acknowledgements}

We thank Ms. Yoshiko Kashiwagi, Ms. Satoko Ohtawa, and Ms. Yasuko Taniji for their skilled technical assistance. This work was supported by the Programme for Promotion of Basic and Applied Researches for Innovations in Bio-oriented Industry from the Biooriented Technology Research Advancement Institution.

\section{References}

Aida R, Shibata M (1995) Agrobacterium-mediated transformation of torenia (Torenia fournieri). Breed Sci 45: 71-74

Aida R, Shibata M (2001) Transgenic Torenia fournieri Lind. (torenia). In: Bajaj YPS (ed) Transgenic Crops III vol 48, Biotechnology in Agriculture and Forestry. Springer Verlag, Berlin, pp 294-305

Aida R (2008) Torenia fournieri (torenia) as a model plant for transgenic studies. Plant Biotechnol 25: 541-545

Bae H, Herman E, Bailey B, Bae HJ, Sicher R (2005) Exogenous trehalose alters Arabidopsis transcripts involved in cell wall modification, abiotic stress, nitrogen metabolism, and plant defense. Physiol Plant 125: 114-126

Iordachescu M, Imai R (2008) Trehalose biosynthesis in response to abiotic stresses. J Integr Plant Biol 50: 1223-1229

Liu THA, Lin JJ, Wu RY (2006) The effects of using trehalose as a carbon source on the proliferation of Phalaenopsis and Doritaenopsis protocorm-like-bodies. Plant Cell Tissue Organ Cult 86: 125-129

Llorente BE, Juarez LM, Apo'stolo NM (2007) Exogenous trehalose affects morphogenesis in vitro of jojoba. Plant Cell Tissue Organ Cult 89: 193-201

Murashige T, Skoog F (1962) A revised medium for rapid growth and bio-assays with tobacco tissue culture. Physiol Plant 15:
473-491

Narumi T, Aida R, Niki T, Nishijima T, Mitsuda N, Hiratsu K, Ohme-Takagi M, Ohtsubo N (2008) Chimeric AGAMOUS repressor induces serrated petal phenotype in Torenia fournieri similar to that induced by cytokinin application. Plant Biotechnol 25: 45-53

Narumi T, Aida R, Koyama T, Yamaguchi H, Sasaki K, Shikata M, Nakayama M, Ohme-Takagi M, Ohtsubo N (2011) Arabidopsis chimeric TCP3 repressor produces novel floral traits in Torenia fournieri and Chrysanthemum morifolium. Plant Biotechnol 28: $131-140$

Sasaki K, Aida R, Niki T, Yamaguchi H, Narumi T, Nishijima T, Hayashi Y, Ryuto H, Fukunishi N, Abe T, Ohtsubo N (2008) High-efficiency improvement of transgenic torenia flowers by ion beam irradiation. Plant Biotechnol 25: 81-89

Sasaki K, Aida R, Yamaguchi H, Shikata M, Niki T, Nishijima T, Ohtsubo N (2010) Functional divergence within class B MADSbox genes TfGLO and TfDEF in Torenia fournieri Lind. Mol Genet Genomics 284: 399-414

Sasaki K, Yamaguchi H, Narumi T, Shikata M, Oshima Y, Nakata M, Mitsuda N, Ohme-Takagi M, Ohtsubo N (2011) Utilization of a floral organ-expressing $A P 1$ promoter for generation of new floral traits in Torenia fournieri Lind. Plant Biotechnol 28: $181-188$

Shikata M, Narumi T, Yamaguchi H, Sasaki K, Aida R, Oshima Y, Takiguchi Y, Ohme-Takagi M, Mitsuda N, Ohtsubo N (2011) Efficient production of novel floral traits in torenia by collective transformation with chimeric repressors of Arabidopsis transcription factors. Plant Biotechnol 28: 189-199

Wingler A, Fritzius T, Wiemken A, Boller T, Aeschbacher RA (2000) Trehalose induces the ADP-glucose pyrophosphorylase gene, ApL3, and starch synthesis in Arabidopsis. Plant Physiol 124: $105-114$ 